PALABRAS CLAVE

Condiciones políticas

Condiciones económicas

Democracia

Gobernabilidad

Corrupción política

Participación política

Desarrollo económico

Distribución del ingreso

América Latina

Fernando Calderón

Coordinador regional Informe Sub-

regional de Desarrollo Humano,

Juventud y desarrollo humano

en el Mercosur

œ fernando.calderon@undp.org
REVISTA DE LA CEPAL 96 DICIEMBRE 2008

\section{Una inflexión histórica. Cambio político y situación socioinstitucional en América Latina}

\author{
Fernando Calderón
}

\begin{abstract}
"La 'consistencia' tradicional de América Latina se extingue hoy sin remedio a la vista de todos. (...) Ante ella son, en consecuencia, ineludibles dos interrogantes fundamentales. Primero, ¿cuáles son los soportes de la nueva estructura que está sucediendo a la anterior y que ésta portaba ya en su seno desde los comienzos de su descomposición? Segundo, ¿dónde se encuentra el último fundamento de la prise de conscience que abre con el nuevo ciclo económico
\end{abstract} la fisonomía del futuro inmediato?"

José Medina Echeverría Sociología latinoamericana

A

mérica Latina vive un momento de crisis, inflexión y cambio político, en el cual las relaciones entre institucionalidad y equidad son fundamentales para la gobernabilidad democrática futura. En particular, la crisis y la inflexión son, ante todo, socioculturales. La política y los actores políticos no estarían en condiciones de resolver los problemas político-institucionales a menos que fuesen capaces de enfrentar los problemas de la sociedad, particularmente aquellos referidos a la equidad y a la pobreza. Este artículo sintetiza y examina un conjunto de tendencias sociohistóricas, formula reflexiones que pretenden ilustrar y desagregar sus principales hallazgos, plantea perspectivas de mediano plazo y propone pautas para mejorar la gobernabilidad democrática en América Latina. 


\section{I}

\section{Introducción}

Este trabajo busca sintetizar las tendencias sociohistóricas de América Latina, que afectan de manera determinante los escenarios de gobernabilidad democrática, y reflexionar sobre ellas.

Luego de un período de importantes reformas estructurales y transición democrática, América Latina inicia un nuevo ciclo histórico. Esto se observa particularmente en los países que integran el Proyecto Regional de Análisis Político y Escenarios de Corto y Mediano Plazo (PAPEP) y que aquí son objeto de estudio. ${ }^{1} \mathrm{La}$ experiencia vivida ha causado un clima de desencanto y malestar, pero también de grandes expectativas de cambio y progreso dentro de un régimen democrático que la sociedad sigue percibiendo como la forma más aceptable de gobierno. El malestar convive con la generación de una ciudadanía más autónoma y crítica, vinculada a nuevos espacios públicos de participación, conflicto y diálogo.

La idea que organiza este trabajo es que América Latina pasa por un momento de crisis, inflexión y cambio político, en el que las relaciones entre institucionalidad y equidad son fundamentales para la gobernabilidad democrática futura. En gran medida, de cómo se avance en ambos planos —enmarcados en contextos económicos internacionales y nacionales específicosdependerán las posibilidades de que el momento de inflexión se transforme en una ruta renovadora de la democracia y el desarrollo.

Se postula asimismo que la crisis y la inflexión son primordialmente socioculturales: la política y los actores políticos no estarán en condiciones de resolver los problemas político-institucionales si no son capaces de encarar los problemas de la sociedad, particularmente aquellos referidos a la equidad y a la pobreza.

Una pregunta fundamental es cómo construir opciones político-institucionales que resuelvan esos problemas. $\mathrm{O}$, dicho de otra manera, cómo - en un contexto de acelerada globalización - la política y las instituciones de la democracia procesarán los cambios que vienen experimentando las sociedades latinoamericanas.

En este artículo se examinan tendencias sociohistóricas y se ofrecen reflexiones que pretenden ilustrar y desagregar sus principales hallazgos. También se plantean perspectivas de mediano plazo bajo la forma de escenarios básicos. Asimismo formula una propuesta para mejorar la gobernabilidad en América Latina, que contiene algunas pautas básicas sobre los aspectos en los que debe hacerse mayor hincapié.

\section{II}

\section{Problemas y desafíos sociohistóricos}

Entre la variedad de problemas y desafíos que enfrentan los distintos países latinoamericanos se han detectado algunos que afectan la gobernabilidad democrática y que constituyen marcos de referencia para los escenarios políticos de corto y mediano plazo. A continuación se examinan los principales. $\square$ Este estudio contó con la colaboración de Juan Enrique Vega y Caterina Colombo. El autor agradece muy especialmente los comentarios y sugerencias al texto preliminar formulados por Fernando Henrique Cardoso, Manuel Castells, Juan Gabriel Valdés y los colegas de la Dirección Regional de América Latina y el Caribe, del Programa de las Naciones Unidas para el Desarrollo (PNUD). Versiones previas fueron presentadas en seminarios internos PAPEP-PNUD. El autor agradece también la cooperación de Diego Achard, Antonio Araníbar, Luis Eduardo González, Armando Ortuño, Gerardo Noto, Eugenia Bóveda y Emilio Sampietro. El presente trabajo ha tomado en consideración estudios nacionales sobre coyuntura y escenarios de corto plazo de Argentina, Bolivia, Ecuador, Honduras, Panamá, Perú, Nicaragua y Uruguay, así como estudios del Observatorio Regional del PAPEP, una amplia bibliografía y datos secundarios sobre el tema.

${ }^{1}$ El PAPEP se enmarca en la estrategia regional del PNUD sobre gobernabilidad democrática en América Latina y busca contribuir al fortalecimiento de esa gobernabilidad. Entre las actividades del Proyecto destaca la elaboración de informes de coyuntura y de escenarios de corto y mediano plazo (véase más información en http:// www.papep.org). 


\section{Las situaciones de crisis y los riesgos político- institucionales}

Las situaciones de crisis y los riesgos político-institucionales que ha venido experimentando buena parte de los países latinoamericanos afectan la evolución democrática, están modificando el perfil político de la región y plantean nuevas formas de vinculación entre las instituciones y la sociedad.

En lo corrido del siglo actual, más del $30 \%$ de las democracias latinoamericanas experimentó crisis políticoinstitucionales agudas que afectaron sus instituciones centrales (presidencia y legislatura). ${ }^{2}$ En conjunto, estas situaciones de crisis aguda y de riesgo o de cuasi crisis ponen de manifiesto que la consolidación de las democracias latinoamericanas es un proceso difícil cuyo éxito no está asegurado. Por lo demás, varios de los regímenes democráticos más consolidados de América Latina están pasando por procesos de inflexión sociopolítica "suave". 3

Un dato empírico que es clave en la relación entre instituciones y sociedad está dado por las expectativas insatisfechas de la población, las que sumadas a instituciones públicas frágiles y condiciones económicas precarias pueden desencadenar episodios de ingobernabilidad. ${ }^{4}$ Tales situaciones revelan las debilidades de las democracias latinoamericanas y los riesgos que

\footnotetext{
${ }^{2}$ Desde el inicio del siglo XXI al menos seis países han vivido crisis de esta naturaleza: Paraguay (2000), Perú (2000), Argentina (2001), Venezuela (2003), Bolivia (2003 y 2005) y Ecuador (2000 y 2005). De las ocho situaciones mencionadas, seis concluyeron con la caída de los respectivos presidentes (Fujimori en Perú, De la Rúa en Argentina, Sánchez de Losada y Mesa en Bolivia y Mahuad y Gutiérrez en Ecuador), ya sea porque la legislatura los destituyó o porque renunciaron; una, con participación militar, culminó con la destitución de Cubas Grau como presidente de Paraguay e incluyó el asesinato de un vicepresidente, y la última fue un golpe de Estado fracasado contra el presidente venezolano Chávez. Hubo otros casos de crisis políticas que se acercaron a situaciones extremas como las mencionadas, pero sin llegar a un momento de ruptura. Entre ellas habría que mencionar la de Nicaragua (fines del 2004 y principios del 2005) y la de Honduras (principios del 2006).

${ }^{3}$ Tal es el caso de Uruguay. Como afirma Moreira (2006), Uruguay no se encuentra en una situación de crisis; sin embargo, "la llegada de la izquierda al gobierno marca un punto de inflexión que, al igual que las crisis, significa una excelente ventana de oportunidad para la búsqueda de un debate público que ponga en común nuestras distintas visiones y perspectivas sobre el país".

${ }^{4}$ Un análisis de González (2006) demuestra empíricamente la relación entre las fragilidades institucionales (índice de efectividad judicial-legal) y las expectativas insatisfechas (crecimiento del PIB per cápita 1975-2003), comprobando una asociación moderada pero significativa entre las dos variables señaladas. Los siete países que experimentaron crisis políticas agudas muestran registros negativos en ambas variables, mientras que los tres países "consolidados" (Chile, Costa Rica y Uruguay) se hallan en el extremo opuesto.
}

enfrentan y que probablemente seguirán enfrentando. Para contribuir a la consolidación de la democracia en América Latina es necesario entonces entender mejor la naturaleza del cambio político y las perspectivas que genera, para buscar soluciones que ayuden a avanzar en el desarrollo democrático.

\section{Los límites de los sistemas de representación e intermediación}

Los problemas político-institucionales están asociados a los límites de los sistemas de representación y a una desconfianza generalizada frente a las instituciones, particularmente los partidos políticos y en general los mecanismos de intermediación (gráfico 1). El surgimiento de un ciudadano autónomo, crítico y reflexivo, así como las demandas de participación local, de reconocimiento de los movimientos indígenas y de equidad de género, son factores que pueden reconfigurar los sistemas de representación.

Los problemas institucionales se relacionan con la débil capacidad de acción política de los partidos para representar institucionalmente a la sociedad. Esto se traduce en crisis de representación e intermediación políticas. La consigna “iQue se vayan todos!” que se escuchó en Argentina, la crisis de octubre del 2003 en Bolivia y las demandas de la llamada Rebelión de los Forajidos en Ecuador son ejemplos del desencanto generalizado con la política y los partidos políticos en la región. Según el Informe Latinobarómetro $2006,{ }^{5}$ un $58 \%$ de los latinoamericanos considera que no puede haber democracia sin partidos políticos, pero solo el $22 \%$ confía en ellos.

En algunos países la crisis de representación se torna más compleja por las expresiones multiculturales, especialmente de los denominados "pueblos originarios", dada la histórica incapacidad de los sistemas institucionales para representarlos. Las demandas de participación y cambio que plantean estos pueblos recorren gran parte de América Latina y están modificando significativamente el sistema político, sobre todo en Bolivia, Ecuador, Perú y Guatemala.

También se observa en muchos países latinoamericanos la persistencia de los límites institucionales a la participación femenina. La desigualdad de género es producto de un conjunto de desigualdades culturales y

\footnotetext{
${ }^{5}$ El Informe Latinobarómetro está registrado en la bibliografía de este artículo por el nombre de la entidad que lo publica, la Corporación Latinobarómetro.
} 


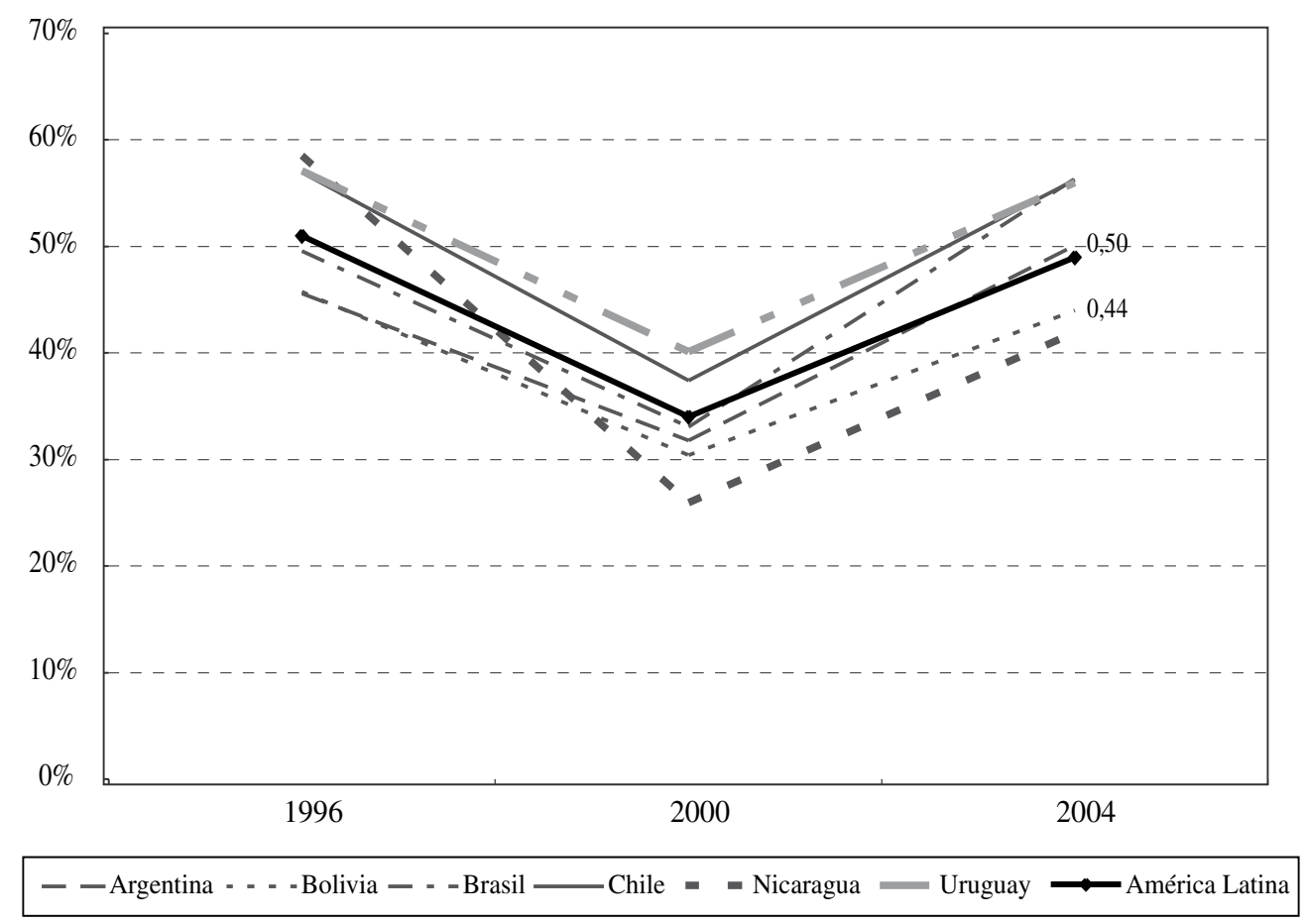

Fuente: elaboración propia con datos de la Corporación Latinobarómetro (varios años).

políticas que se refuerzan mutuamente. Estas condicionan el acceso de las mujeres a derechos de propiedad, riqueza y educación, restringiendo su ingreso a los mercados laborales y, en general, a las esferas de poder. A pesar de los avances registrados por las mujeres en materia de políticas públicas y participación política, ${ }^{6}$ los actores político-institucionales aún tienen dificultades para procesar los cambios y las demandas en función de la equidad de género. Esto tiene efectos adversos sobre la calidad de la democracia y la gobernabilidad democrática.

Por último, en América Latina parecen profundizarse las divisiones entre regiones subnacionales (territorios).

\footnotetext{
${ }^{6}$ Los datos empíricos muestran que la participación política de la mujer en los países en estudio se ha incrementado, aunque sigue siendo baja. Si bien en Argentina, Bolivia, Brasil, Ecuador, Honduras, México, Panamá y Perú se han establecido cuotas que determinan un nivel mínimo de representación femenina —entre $20 \%$ y $40 \%$ - en las listas partidarias de elecciones legislativas, solo en Argentina la participación de la mujer en el Parlamento es superior al 30\%. En los demás países el porcentaje oscila entre el 23\%, en México, y el 6\%, en Honduras (CEPAL, 2006a).
}

Se están generando nuevos conflictos y oposiciones entre lo global, lo local y lo nacional. Hoy las regiones subnacionales buscan formas propias de legitimidad democrática, autonomía en el ámbito nacional y conexión directa con la globalización; estas demandas y aspiraciones se traducen en tensiones económicas, con manifestaciones políticas y electorales, y a veces en grandes crisis político-institucionales. Este fenómeno se observa claramente en Bolivia - entre el altiplano y las tierras bajas-, cuyas elites consideran que los factores regionales subnacionales se hallan entre los principales componentes de la crisis. Pero en muchos otros países se observan fracturas subnacionales (costa-sierra o norte-sur) que se expresan políticamente. En este plano también hay tensiones por el surgimiento de un nuevo orden institucional.

\section{Demandas de más participación del Estado y de mayor control ciudadano}

De distinta manera y con diferente intensidad se han instalado en el espacio político, por una parte, demandas de 
mayor participación del Estado en la gestión del desarrollo y la expansión de la institucionalidad democrática y, por otra, demandas de mayor control ciudadano sobre el poder público.

Buena parte de la población aboga por una nueva relación entre el Estado y la economía, en la que el Estado genere integración social y equidad pero a la vez potencie la economía en el marco de la globalización. Según el Informe Latinobarómetro 2005, solo un promedio de $31 \%$ de los latinoamericanos entrevistados considera que las privatizaciones fueron provechosas. Por su parte, en el Informe Latinobarómetro 2006 se indagó en la responsabilidad por la producción de riqueza. Para tal efecto se utilizó una escala de 1 a 10, en la que " $1=$ el Estado tiene que producir la riqueza" y " $10=$ los privados tienen que producir la riqueza"; se advirtió un grado de estatismo medianamente alto entre los latinoamericanos, ya que el índice medio de la región fue de 4,1. En Argentina, según entrevistas realizadas a las elites y encuestas de opinión pública, existe el deseo compartido de que se redefinan las relaciones entre las empresas y el Estado. En efecto, el 28,5\% de los encuestados afirmó que el Estado debe intervenir para garantizar una justa distribución de los beneficios del crecimiento económico; el 45,6\% expresó el deseo de que las empresas de servicios públicos vuelvan a ser estatales y el $44,7 \%$, de que sigan siendo privadas pero con mayor control estatal (PNUD Argentina, 2005). De manera similar, más del $70 \%$ de los bolivianos manifestó su deseo de que el Estado tenga mayor participación en el negocio del gas (PNUD Bolivia, 2004). En general, todos los estudios nacionales realizados rescatan la idea de fortalecer la "estatalidad" para reforzar la gobernabilidad democrática.

También es probable que esta demanda de mayor intervención estatal se relacione, por una parte, con la necesidad de más seguridad humana que afecta de manera creciente la vida cotidiana de los ciudadanos ${ }^{7}$ $\mathrm{y}$, por otra, con el aumento de acciones anómicas por parte de grupos sociales excluidos, especialmente de jóvenes, que reflejan los límites de la cohesión social y cierto grado de fractura social.

Por otro lado, hay demandas de coordinación entre la ciudadanía y el Estado para propiciar la incorporación de mecanismos que permitan una mayor participación

\footnotetext{
${ }^{7}$ De acuerdo con datos de la Organización Mundial de la Salud (OMS), la tasa de homicidios por cada 100.000 habitantes en 1991 era de 16,7, mientras que en el 2000 ascendía a 27,5. Información disponible en http://www.who.int/violence_injury_prevention/violence/ world_report/en/annex.pdf.
}

CUADRO 1

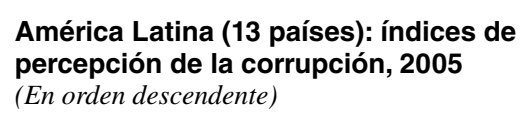

\begin{tabular}{lcc}
\hline País & Posición & Índice \\
\hline Chile & 21 & 7,3 \\
Uruguay & 32 & 5,9 \\
Colombia & 55 & 4,0 \\
Brasil & 62 & 3,7 \\
México & 65 & 3,5 \\
Panamá & 65 & 3,5 \\
Perú & 65 & 3,5 \\
Argentina & 97 & 2,8 \\
Honduras & 107 & 2,6 \\
Nicaragua & 107 & 2,6 \\
Bolivia & 117 & 2,5 \\
Ecuador & 117 & 2,5 \\
Guatemala & 117 & 2,5 \\
\hline
\end{tabular}

Fuente: elaboración propia con datos de Transparencia Internacional.

ciudadana, así como demandas de consolidación de las instituciones del Estado, con un sentido público y de unidad estatal. En unas y otras ronda el tema de la rendición de cuentas (accountability), que tiene que ver con la transparencia, la información y los mecanismos de control ciudadano en lo que se refiere a las políticas públicas. Las demandas de un poder judicial transparente, autónomo e institucional revelan que este constituye un elemento de referencia muy importante para la población, como lo refleja claramente el índice de percepción de la corrupción que elabora Transparencia Internacional (cuadro1).

\section{Los problemas de desigualdad y pobreza}

Estos problemas están en el centro de las tendencias socioeconómicas y pueden constituir los factores que más inciden tanto en la gestación de nuevas opciones políticas como en la gobernabilidad democrática de los países estudiados.

Las políticas de reforma estructural aplicadas por los países de América generaron un conjunto de transformaciones socioeconómicas que modificaron la conformación de las diversas sociedades nacionales. Uno de sus efectos más significativos fue el de hacer cada vez más complejos los procesos de diferenciación social, lo que ha afectado la calidad de las formas de reproducción social.

Si bien los procesos de diferenciación social se han traducido en un incremento de las disparidades entre los países en desarrollo y los industrializados - América Latina sigue siendo una de las regiones con mayor 
desigualdad en el mundo e incluso viene incrementando las desigualdades y diferencias entre naciones-, los países en desarrollo se caracterizan porque en ellos ha persistido o aumentado la mayoría de las brechas internas. En muchos creció la participación del 20\% más rico de la población en los ingresos, mientras que la participación del $20 \%$ más pobre se mantuvo constante o disminuyó (cuadro 2). ${ }^{8}$ Diversos indicadores sociales también muestran profundas disparidades: de género, entre regiones subnacionales, socioeconómicas y étnicas.

En cuanto a las disparidades de género, persisten importantes diferencias salariales entre varones y mujeres con la misma cantidad de años de estudio aprobados (cuadro 3). En promedio, las mujeres ganan aproximadamente el $80 \%$ de lo que ganan los varones. Los efectos

CUADRO 2

\section{América Latina (13 países): ${ }^{a}$ concentración del ingreso de las personas, 1990 y 2005 (En porcentajes)}

\begin{tabular}{|c|c|c|c|c|c|c|}
\hline \multirow{2}{*}{ País } & \multicolumn{3}{|c|}{$\begin{array}{l}20 \% \text { más pobre } \\
\text { de la población }\end{array}$} & \multicolumn{3}{|c|}{$\begin{array}{l}20 \% \text { más rico } \\
\text { de la población }\end{array}$} \\
\hline & 1990 & 2005 & Variación & 1990 & 2005 & Variación \\
\hline Argentina $^{\mathrm{b}}$ & 4,1 & 3,5 & $-0,6$ & 54,9 & 57,2 & 2,3 \\
\hline Bolivia & 1,3 & 1,5 & 0,2 & 61,2 & 64,4 & 3,2 \\
\hline Brasil & 2,1 & 2,4 & 0,3 & 66,8 & 65,5 & $-1,3$ \\
\hline Chile & 3,5 & 3,7 & 0,2 & 60,6 & 60,4 & $-0,2$ \\
\hline Colombia & 3,7 & 2,9 & $-0,8$ & 57,8 & 63,0 & 5,2 \\
\hline Ecuador $^{\mathrm{b}}$ & 4,8 & 3,8 & $-1,0$ & 51,9 & 56,3 & 4,4 \\
\hline Guatemala $^{c}$ & 2,7 & 3,7 & 1,0 & 62,7 & 59,3 & $-3,4$ \\
\hline Honduras & 2,4 & 2,4 & 0,0 & 65,7 & 62,4 & $-3,3$ \\
\hline México & 3,9 & 3,7 & $-0,2$ & 58,7 & 57,6 & $-1,1$ \\
\hline Nicaragua & 2,1 & 2,5 & 0,4 & 61,3 & 61,7 & 0,4 \\
\hline Panamá & 2,8 & 2,5 & $-0,3$ & 60,0 & 58,2 & $-1,8$ \\
\hline Perúd $^{\mathrm{d}}$ & 3,0 & 3,8 & 0,8 & 57,9 & 55,2 & $-2,7$ \\
\hline Uruguay $^{b}$ & 5,1 & 4,8 & $-0,3$ & 51,9 & 50,7 & $-1,2$ \\
\hline
\end{tabular}

Fuente: elaboración propia con datos de CEPAL (2007).

a Criterio de selección: se incluyen los países que integran el PAPEP (Argentina, Bolivia, Ecuador, Honduras, Nicaragua, Panamá, Perú y Uruguay) y otros que se consideraron pertinentes para referencia y comparación.

b Zonas urbanas.

c Los datos corresponden a 1990 y 2002.

d Los datos corresponden a 1997 y 2003.

\footnotetext{
${ }^{8}$ Entre 1990 y el 2005 el coeficiente de Gini disminuyó en Brasil, Colombia, Guatemala, Honduras, México y Uruguay, se mantuvo invariable en Chile, Nicaragua y Panamá y aumentó en Argentina, Bolivia y Ecuador. Más aún, en el 2005 solamente Uruguay puede incluirse entre los países con baja desigualdad, en tanto que gran parte de los países de la región exhibe altos niveles de desigualdad, y en el caso de Brasil, Argentina y Honduras, esos niveles son muy altos (CEPAL, 2007).
}

CUADRO 3

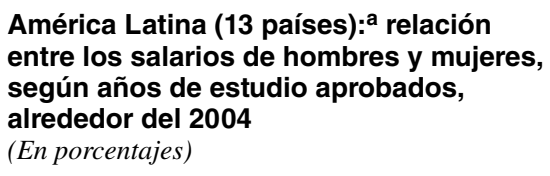

\begin{tabular}{lccc}
\hline País & Año & Total & $\begin{array}{c}\text { 13 y más años } \\
\text { de educación }\end{array}$ \\
\hline Argentina & 2004 & 86,6 & 76,6 \\
Bolivia & 2002 & 74,4 & 64,2 \\
Brasil & 2003 & 76,7 & 61,9 \\
Chile & 2003 & 81,7 & 69,0 \\
Colombia & 2002 & 89,2 & 80,0 \\
Ecuador & 2002 & 83,7 & 72,9 \\
Guatemala & 2002 & 82,4 & 87,0 \\
Honduras & 2002 & 82,8 & 68,7 \\
México & 2004 & 82,1 & 69,6 \\
Nicaragua & 2001 & 83,0 & 71,9 \\
Panamá & 2002 & 82,7 & 71,4 \\
Perú & 2003 & 77,4 & 70,8 \\
Uruguay & 2002 & 80,8 & 66,8 \\
\hline
\end{tabular}

Fuente: elaboración propia con datos de CEPAL (2006a).

a Criterio de selección: se incluyen los países que integran el PAPEP (Argentina, Bolivia, Ecuador, Honduras, Nicaragua, Panamá, Perú y Uruguay) y otros que se consideran pertinentes para referencia y comparación.

adversos de esta desigualdad salarial pueden agudizarse por el incremento del número de jefas de hogar.

La diferenciación social también se advierte en las considerables disparidades entre países en la proporción de indigentes, lo que tiene efectos significativos en la gobernabilidad. En un extremo (Bolivia, Guatemala, Honduras y Nicaragua), la indigencia afecta a más del $30 \%$ de la población total, mientras que en el otro (Chile, Costa Rica y Uruguay) los niveles de indigencia son inferiores al 10\% (CEPAL, 2007).

Cabe señalar que la pobreza en los países latinoamericanos no es homogénea, sino diferenciada social y culturalmente, lo que se comprueba al observar la desigual proporción de pobres por zona geográfica, género y origen étnico. En el 2005, el 39,8\% de los latinoamericanos se encontraba por debajo de la línea de pobreza, pero al considerar exclusivamente las zonas rurales este porcentaje aumentaba al 58,8\% (gráfico 2).

Además, como señalan Buvinic y Roza (2004), el género y el origen étnico interactúan y generan un grado de exclusión social aún mayor. Como consecuencia, los niveles educativos de las mujeres indígenas son mucho más bajos que los de las mujeres no indígenas. Más del $50 \%$ de las niñas indígenas de Bolivia y Guatemala han abandonado el colegio a los 14 años (Arias y Duryea, 2003). En Bolivia, Perú y Guatemala, las mujeres 
GRÁFICO 2
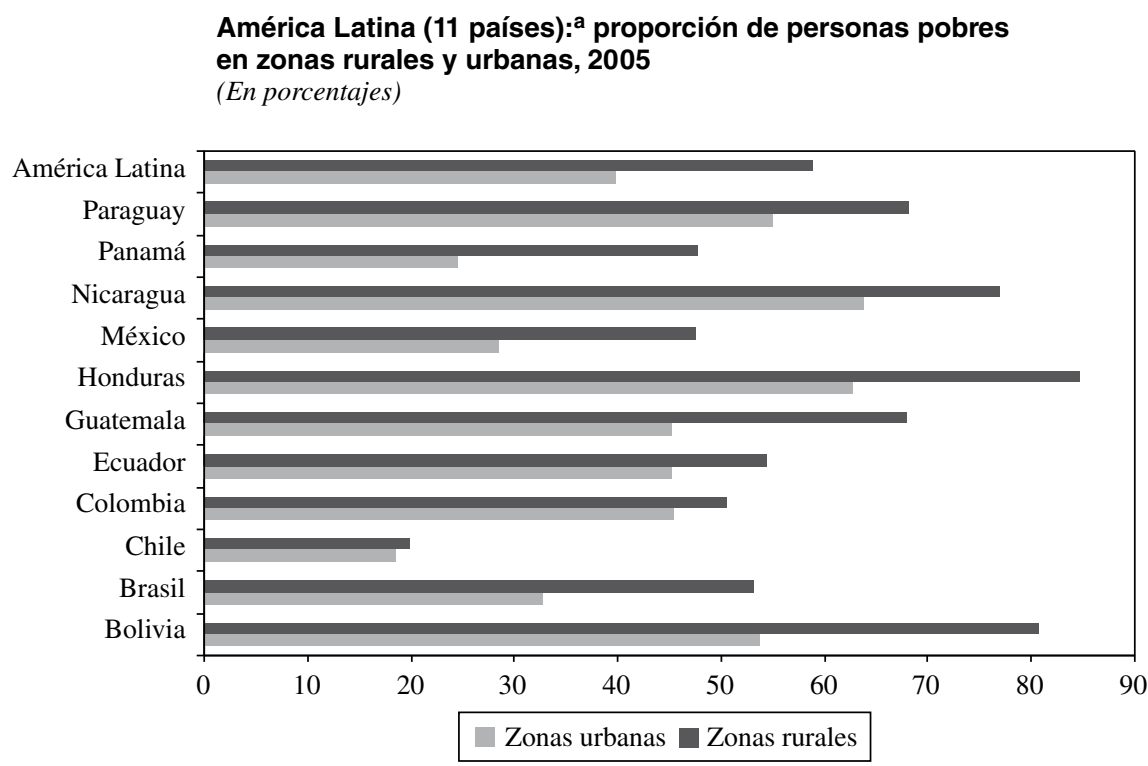

Fuente: elaboración propia con datos de CEPAL (2007).

a Criterio de selección: se incluyen los países que integran el PAPEP (Argentina, Bolivia, Ecuador, Honduras, Nicaragua, Panamá, Perú y Uruguay) y otros que se consideran pertinentes para referencia y comparación.

indígenas tienen mayores probabilidades de obtener empleos de baja remuneración y en el sector informal (Duryea y Genoni, 2004). A la interacción adversa señalada se agrega otro factor, la zona geográfica, ya que los pueblos indígenas suelen habitar zonas rurales que presentan niveles de pobreza superiores a los de las áreas urbanas.

Para ser eficaces, la gobernabilidad y las políticas públicas dirigidas a combatir la pobreza deben tener en cuenta cuán complejas y heterogéneas son la desigualdad y la pobreza misma. La relación entre gobernabilidad y desigualdad social también está asociada con las percepciones subjetivas de las personas, con la disposición social a cambiar la situación, con la frustración de las expectativas de una población más educada, con el comportamiento limitado de las elites, en el sentido de que no alcanzan a dirigir sus sociedades y solo las dominan, y, muy especialmente, con la dinámica de los viejos y nuevos conflictos socioculturales. Enfrentar la cuestión de la pobreza y la inequidad significa sobre todo potenciar la acción de las sociedades y los Estados para generar políticas que fortalezcan las capacidades ciudadanas de los más pobres y excluidos. Por su parte, los mismos pobres y/o discriminados estarían buscando transformar sus necesidades en demandas que se expresen en el siste- ma político y que generen resultados. Lo que estaría en juego entonces en la gestión de la gobernabilidad democrática sería la capacidad de gestión del cambio que ya está en curso.

\section{Frustración de expectativas en la población}

Un factor crucial de la relación entre cambio políticoinstitucional y cambio social es una creciente frustración de las expectativas de la población, que responde a los graves problemas de desigualdad y pobreza descritos, unidos a niveles de educación más altos y mayor acceso a los medios de comunicación tradicionales y nuevos (cuadro 4). El resultado son sociedades más expuestas al consumo cultural y con mayor educación, pero sin los medios para satisfacer las demandas que tal exposición origina. Este fenómeno genera frustración de expectativas y probablemente es una de las principales fuentes del conflicto y malestar que dificultan la gobernabilidad democrática.

En este contexto, la ciudadanía ya no se satisface con las ofertas políticas (que se perciben como incumplidas) ni con las opciones de una prometedora economía de mercado. Los ciudadanos tienden a ser más realistas, críticos e informados y poseen una visión más clara de su autonomía y libertad de acción. 


\begin{tabular}{|c|c|c|c|c|c|c|c|c|c|}
\hline \multirow{2}{*}{ País } & \multicolumn{3}{|c|}{$\begin{array}{l}\text { Líneas telefónicas y } \\
\text { teléfonos móviles }\end{array}$} & \multicolumn{3}{|c|}{ Computadores personales } & \multicolumn{3}{|c|}{ Usuarios de Internet } \\
\hline & 1990 & 2004 & Variación & 1998 & 2004 & Variación & 1996 & 2004 & Variación \\
\hline Argentina & 9,3 & 58,1 & 48,8 & 5,5 & 8,2 & 2,7 & 0,2 & 16,1 & 15,9 \\
\hline Bolivia & 2,8 & 27,0 & 24,2 & 0,8 & 2,3 & 1,5 & 0,2 & 3,9 & 3,7 \\
\hline Brasil & 6,5 & 59,8 & 53,3 & 3,0 & 1,7 & 7,7 & 0,5 & 12,2 & 11,7 \\
\hline Chile & 6,7 & 83,6 & 76,9 & 6,3 & 13,9 & 7,6 & 0,7 & 27,9 & 27,2 \\
\hline Colombia & 6,5 & 40,1 & 33,6 & 3,0 & 5,5 & 2,5 & 0,5 & 8,9 & 8,4 \\
\hline Ecuador & 4,8 & 39,1 & 34,3 & 1,9 & 5,5 & 3,6 & 0,1 & 4,7 & 4,6 \\
\hline Guatemala & 2,1 & 34,0 & 31,9 & 0,8 & 1,8 & 1,0 & 0,0 & 6,0 & 6,0 \\
\hline Honduras & 1,7 & 15,7 & 14,0 & 0,8 & 1,6 & 0,8 & 0,0 & 3,2 & 3,2 \\
\hline México & 6,6 & 53,9 & 47,3 & 3,7 & 10,7 & 7,0 & 0,2 & 13,4 & 13,2 \\
\hline Nicaragua & 1,3 & 16,8 & 15,5 & 1,9 & 3,5 & 1,6 & 0,1 & 2,2 & 2,1 \\
\hline Panamá & 9,3 & 38,8 & 29,5 & 2,7 & 4,1 & 1,4 & 0,2 & 9,5 & 9,3 \\
\hline Perú & 2,6 & 22,1 & 19,5 & 3,0 & 9,7 & 6,7 & 0,3 & 11,6 & 11,3 \\
\hline Uruguay & 13,4 & 49,4 & 36,0 & 9,1 & 13,3 & 4,2 & 1,9 & 21,0 & 19,1 \\
\hline América Latina & 6,4 & 50,1 & 43,7 & 3,4 & 9,1 & 5,8 & 0,3 & 11,9 & 11,6 \\
\hline
\end{tabular}

Fuente: elaboración propia con datos de CEPAL (2007).

a Criterio de selección: se incluyen los países que integran el PAPEP (Argentina, Bolivia, Ecuador, Honduras, Nicaragua, Panamá, Perú y Uruguay) y otros que se consideran pertinentes para referencia y comparación.

\section{Liderazgo débil de las elites dirigentes}

Un elemento que contribuye a las crisis institucionales es el débil liderazgo nacional de las elites dirigentes.

Pareciera que los cambios y las limitaciones estructurales del liderazgo social y político, particularmente de los grupos de poder, inciden en el bajo perfil institucional de la mayoría de los países latinoamericanos. Cabe preguntarse entonces cuál es la capacidad de las elites para articular una visión y una práctica nacional dirigidas a resolver los problemas socioinstitucionales en los marcos de una gobernabilidad global.

Por otra parte, la percepción de las elites es de especial relevancia para explorar la evolución de la gobernabilidad democrática. Las entrevistas realizadas por el PAPEP a 206 líderes empresariales, políticos y sociales revelan que en esos grupos existe un consenso generalizado de que los problemas de pobreza y de institucionalidad constituyen los principales desafíos que enfrenta la democracia en América Latina. La información obtenida por el Observatorio Regional PAPEP indica que: i) alrededor del 50\% de las elites de Argentina, Brasil y México considera que la pobreza y la inequidad son las causas recurrentes de los problemas de la democracia en la región; ii) la mayoría de las elites europeas cree que el principal problema de la región es la mala distribución del ingreso y la pobreza estructural, y iii) las elites de los Estados Unidos tienen opiniones divididas, ya que el $47 \%$ cree que el principal desafío de América Latina son las falencias institucionales y el $44 \%$, que lo son la pobreza y la desigualdad (Observatorio Regional PAPEP, 2006-2007).

La opinión pública coincide con las elites en considerar que la pobreza y la desigualdad afectan el funcionamiento de la democracia en América Latina. Según el Informe Latinobarómetro 2005, en una escala de 1 (los más pobres) a 10 (los más ricos), la autocalificación que se dan los latinoamericanos es en promedio de 3,66. En el Informe Latinobarómetro 2006 se señala que más del $66 \%$ de los latinoamericanos considera que los países son gobernados por unos pocos grupos poderosos que solo buscan su beneficio personal. Más aún, en Ecuador, Guatemala, Nicaragua y Panamá menos del 20\% de sus habitantes cree que se gobierna para beneficiar a toda la población. Por su parte, las elites de Argentina están sintonizadas solo parcialmente con la percepción de los ciudadanos ya que, a diferencia de estos, muestran cierta renuencia a valorar la evolución de los años recientes y priorizar la 
lucha contra la pobreza. ${ }^{9}$ En Honduras, el desprestigio de las elites afecta particularmente a los jueces, en quienes no confía el $51 \%$ de los hondureños, y a los diputados del Congreso Nacional, de quienes desconfía el 71\% de la población (PNUd Honduras, 2005).

Parecería que en América Latina persisten orientaciones culturales que han sido tradicionales de las elites — referidas a falta de austeridad y de solidaridad y a escasa articulación entre fines y medios- que explicarían la distribución desigual del poder y las percepciones de la opinión pública. Sin embargo, también ha habido cambios en el patrón de comportamiento de las elites, o han emergido nuevas elites con orientaciones culturales diferentes. Un mayor conocimiento de las modificaciones en la composición, las formas de socialización y las orientaciones de las elites ayudaría a construir mejor el mapa político, factor clave para diagnosticar las tendencias de la gobernabilidad democrática en la región.

\section{Aumento y fragmentación de los conflictos sociales}

En términos generales, los conflictos sociales en las sociedades latinoamericanas han aumentado y se han hecho más intensos, pero también se han fragmentado. Se trata en algunos casos de conflictos de reacción y protesta y en otros de nuevas fuerzas socioculturales que configuran nuevas opciones políticas. Estos conflictos ponen de manifiesto las dificultades de las instituciones y del Estado para lograr integración, reconocimiento y progreso social. De alguna manera se están gestando nuevos mapas políticos que no tienen un único referente de orientación sino varios, intensos y limitados. Se puede decir entonces que estas sociedades son policéntricas.

Los ciudadanos han buscado otros canales para expresar sus demandas y su malestar con la política, tanto a través de organizaciones alternativas con capacidad de cuestionamiento y protesta que debilitan al Estado, como a través de movilizaciones en las calles. Varias de estas movilizaciones confluyeron en proyectos políticos alternativos, como el del Movimiento al Socialismo (MAS) en Bolivia, o en apoyo a fuerzas progresistas en varios países del continente. También se observan conductas

\footnotetext{
${ }^{9}$ Solo $17 \%$ de los empresarios considera que la pobreza es el principal problema del país, mientras que $30,2 \%$ de los argentinos encuestados mencionó el desempleo y la pobreza como los dos aspectos que más deben mejorar en el país (PNUD Argentina, 2005).
}

de anomia, sobre todo de los jóvenes, contra un orden que no reconocen ni los integra.

En síntesis, los problemas de institucionalidad y los de inequidad y pobreza se refuerzan mutuamente, además de ser afectados por la situación social preexistente. Esto marcaría con fuerza los límites de la democracia en América Latina y perfilaría escenarios muy difíciles. De ahí la importancia crucial de vincular la reforma social con la reforma político-institucional para mejorar la gobernabilidad democrática

\section{Los espacios de comunicación}

Los espacios de comunicación, sean ellos los tradicionales o las nuevas redes electrónicas, son cada vez más importantes para entender los ámbitos y agendas del poder y el cambio político. El conjunto de los actores políticos y socioculturales participa y toma decisiones en estos espacios y la gobernabilidad no puede ser entendida fuera de ellos.

Un fenómeno de especial relevancia es la mediatización de la política por la televisión y, más recientemente, por los medios de comunicación horizontales, como Internet, la telefonía celular y otros. La arena de expresión o representación de la política es cada día más mediática y los medios de comunicación se caracterizan principalmente por ser los espacios donde se expresa el poder. En la región, según datos del Informe Latinobarómetro 2006 , la televisión y la radio se encuentran entre las instituciones más confiables: 69\% y 64\% de los consultados, respectivamente, afirma confiar en ellas; además, la televisión es el medio de información más utilizado: en 2006, el 83\% de los latinoamericanos vio las noticias en la televisión.

De hecho, en rondas de consultas a 231 líderes de América Latina, un $65 \%$ de ellos ubicó a los medios de comunicación entre los principales poderes fácticos de la región (PNUD, 2004). En Bolivia los medios de comunicación, y en particular la televisión, tienen un impacto creciente en el ejercicio de la política (PNUD Bolivia, 2004). En Ecuador, durante la Rebelión de los Forajidos (abril del 2005), decenas de miles de familias convocadas por correo electrónico y telefonía celular ocuparon las calles de Quito, lo que llevó a la renuncia del Presidente Lucio Gutiérrez. Por lo general, el espacio mediático determina la dinámica de los sistemas de representación política y condiciona la legitimidad institucional en la región; asimismo, plantea el surgimiento de la democracia de lo público, tema que requiere más investigación y análisis. 


\section{III}

\section{Escenarios básicos de gobernabilidad}

La evolución de la economía mundial entre los años 2005 y 2007 fue favorable, en particular para América Latina que tuvo una tasa de crecimiento positiva - aproximadamente 5\% en el 2006-, con una leve desaceleración en el 2007 (CEPAL, 2006b), debido al menor ritmo de la actividad económica mundial, el aumento del precio del petróleo y la incipiente crisis sub prime que desataría durante el año en curso los actuales vientos de recesión mundial. La moderación del crecimiento fue acompañada por una convergencia regional en torno a la tasa de crecimiento media de América Latina y el Caribe. En este sentido, es posible establecer dos categorías de países: i) aquellos con tasas de crecimiento excepcionalmente altas en el 2004 (Argentina y Uruguay), que crecerían a un ritmo más cercano al promedio, y ii) aquellos que ese mismo año tuvieron un ritmo de crecimiento inferior al promedio (Bolivia), ritmo que se aceleraría.

La actividad económica de la región fue impulsada en gran medida por la demanda externa. Al respecto, mejoró para América Latina la relación de precios del intercambio y en medida aún mayor para los países exportadores de petróleo, gas o minerales metálicos (como Bolivia, Chile, Perú y República Bolivariana de Venezuela). En un escenario optimista, se espera que los precios del café y del azúcar se mantengan en los altos niveles actuales, al igual que las remesas que efectúan los migrantes a sus países de origen.

Los escenarios de crecimiento de la economía son relativamente favorables o menos negativos para la región en el mediano plazo. Sin embargo, el crecimiento que se logre no generará automáticamente una mayor equidad social ni se articulará por sí solo adecuadamente con la política y las instituciones. Cabe preguntarse entonces cómo afectará este crecimiento a la dinámica socioinstitucional, de qué manera las orientaciones del crecimiento económico promoverán dinámicas de equidad social y cuál será el rol del Estado nacional.

Las diversas fuerzas políticas están planteando distintos modos de integrar las demandas sociales y la presión popular. En este contexto es posible visualizar cuatro orientaciones políticas generales y tres tipos de escenarios de gobernabilidad.

\section{Orientaciones políticas generales}

Para agrupar tales orientaciones se ha elaborado la siguiente tipología, basada en las características políticas predominantes en la región. ${ }^{10}$

i) Modernización conservadora: modernización del sistema de partidos, retorno y reforzamiento de la autoridad, reforma institucional para vincular actores y sistemas, relegitimación de la autoridad sobre la base de valores indiscutibles y compartidos, orden moral asociado a la expansión del mercado y asociación ideológica con los Estados Unidos.

ii) Reformismo práctico: renovación y recreación del sistema de partidos, orden laico, construcción de alianzas, búsqueda por el Estado de una gestión institucional que combine crecimiento económico con políticas de inclusión, sistema de partidos moderno y relación pragmática con los Estados Unidos.

iii) Nacionalismo popular: búsqueda de hegemonía política, papel central del Estado en la organización del desarrollo y la democracia, movilización de masas, liderazgo carismático legitimado por la democracia, primacía de la redistribución por sobre la producción y antiimperialismo.

iv) Indigenismo neodesarrollista: participación política ampliada y democracia deliberativa, movimientos sociales e indígenas fuertes, Estado que negocia con las empresas transnacionales y busca inclusión; orden igualitario y antiimperialismo moderado.

Dadas estas orientaciones, cabe preguntarse en qué formas se encaran los siguientes interrogantes: ¿Cómo se gestionan las demandas de la sociedad y cómo se distribuye la riqueza? ¿Cómo se opera en condiciones de movilización social creciente, particularmente en países con importantes recursos naturales? ¿Cómo se logra un control político democrático del conjunto del

\footnotetext{
${ }^{10}$ Esta tipología, de carácter cualitativo, se construyó sobre la base de discursos de líderes políticos, programas partidarios y resultados electorales.
} 
aparato estatal? En suma, ¿cómo se combina crecimiento, transformación institucional y distribución del poder?

\section{Escenarios}

En un escenario ideal se lograría una interacción virtuosa entre reformas sociales y transformaciones institucionales, con una alta capacidad de anticipación y de gestión del conflicto; el resultado sería una gobernabilidad progresiva y un dinamismo económico sostenible. En un segundo escenario, de carácter inercial, persistiría un divorcio entre lo social y lo institucional: solo se realizarían las reformas políticas y sociales indispensables para mantener una gobernabilidad económica mínima, y las capacidades de anticipación y gestión de conflictos serían irregulares. En un tercer escenario, se generarían explosiones sociales, fragmentación de conflictos y quiebres institucionales que podrían llegar incluso a situaciones de caos social, crisis económica y regresiones democráticas; la capacidad de anticipación y de gestión del conflicto sería muy baja.

En realidad, los escenarios probables están condicionados por las capacidades políticas de los actores nacionales para articular los cambios internos con la participación en el cambio global. Posiblemente se hallarán cerca de alguno de los tres escenarios señalados, combinando de diferentes maneras institucionalidad, equidad-pobreza y capacidad de innovación de los actores del sistema político. Tal vez ponderarán de distinta manera cada componente y tendrán diversas trayectorias políticas. Algunos privilegiarán los avances en la institucionalidad y buscarán disminuir la pobreza, mientras que otros procurarán mejorar la equidad con escasos avances institucionales. Quizás otros busquen combinaciones sui generis. Así, las combinaciones serán diversas, como resultado de las acciones y las orientaciones políticas prevalecientes en la región.

Los escenarios enfrentan constantemente cambios y riesgo. La forma en que los riesgos sean transformados en oportunidades y en que se pueda vivir la incertidumbre — inherente al cambio moderno - seguramente influirá en la conformación de las trayectorias políticas que pueda asumir la sociedad en cuestión. Las bases institucionales previas y los avances registrados en términos de equidad y de lucha contra la pobreza constituyen otros importantes antecedentes de las trayectorias políticas. En el mismo sentido, la fuerza y la calidad de la participación ciudadana tendrán un influjo crucial en la calidad del cambio. Allí donde se potencie a ciudadanos capaces de optar por alternativas o de construirlas, asumiendo riesgos, mayores serán las posibilidades de que haya una evolución virtuosa. En suma, la calidad de la matriz política que se logre será un factor decisivo.

Los escenarios de mediano plazo que se plantean en diversos estudios nacionales prefiguran las tendencias probables que exhibirán los países de la región que integran el proyecto PAPEP. ${ }^{11}$

\section{IV}

\section{Pautas para una agenda de gobernabilidad democrática}

El momento de inflexión histórica que vive América Latina ofrece nuevas posibilidades de combinar la oportunidad económica con el fortalecimiento de la capacidad de los actores políticos y socioculturales para impulsar un nuevo ciclo de democracia y desarrollo en función de más y mejor equidad social. Las opciones políticas son variadas y el desafío que se enfrenta es comprender los cambios que están ocurriendo y actuar sobre ellos a partir de las nuevas tendencias que se dan en la sociedad, la economía y la política. En todos los países está surgiendo una nueva forma de ciudadanía y han aparecido nuevas formas de vinculación entre sociedad y Estado. Parece probable que haya una redistribución social del poder.

En una agenda para la acción en materia de gobernabilidad democrática cabría incluir los aspectos que se describen a continuación.

i) Recuperar y fortalecer la ética política en función de la equidad y el respeto a las instituciones. Es necesario promover una cultura política que valore el bien público, pero asociada a rigurosas políticas de fiscalización de

${ }^{11}$ Véase información más detallada en www.papep.org. 
la actividad pública. Por lo tanto, es necesario reforzar la fiscalización por los ciudadanos y el desarrollo de instituciones independientes con facultades específicas de investigación judicial, e incluso permitir auditorías de organizaciones internacionales (por ejemplo, de Transparencia Internacional).

ii) Vincular la renovación de la política con los cambios experimentados por la sociedad —nueva estructura social, nuevos mecanismos de reproducción intercultural, nuevos patrones de socialización, desarrollo de la sociedad-red, importancia estratégica de la migración y otros - y también con los nuevos problemas y desafios de la globalización. Esto supone reconstruir la capacidad de gestión política de la sociedad, fortalecer a los actores políticos y socioculturales, mejorar la capacidad de análisis autónomo e independiente (en universidades, centros académicos y otras instituciones) con el fin de elaborar propuestas de corto y mediano plazo, impulsar procesos de deliberación, construir opciones compartidas, establecer nuevas gramáticas del conflicto (es decir, nuevos lenguajes con reglas distintas y más complejas), y orientar la renovación hacia resultados que legitimen la propia acción política. En definitiva, se trata de establecer agendas enfocadas a gestionar la renovación de la política. Ningún partido político posee por sí solo los recursos para dirigir los procesos de desarrollo. La nueva complejidad social exige una coordinación policéntrica, una red de negociaciones y acuerdos, y también avanzar más allá de las democracias puramente electorales, sin que esto signifique desconocer su valor intrínseco. La renovación de los partidos políticos no puede provenir únicamente del interior del sistema de partidos, sino que debe partir de una nueva relación entre Estado y sociedad.

iii) Proponer nuevas formas de representación y participación para recuperar la confianza en el sistema de partidos y en las instituciones de la democracia. Esto significa incluir en el juego institucional los temas de reconocimiento cultural. Dicha inclusión supone reconocer formas históricas de autogobierno y en especial la interculturalidad en todos los ámbitos de la vida pública, así como crear mecanismos institucionales (asambleas indígenas, de regiones subnacionales y otras) con derechos específicos dentro de la estructura institucional del Estado. También implica asumir la representación y deliberación públicas como forma de autoorganización que promueve nuevos canales de participación tanto temáticos como vinculados a regiones subnacionales. Parece fundamental conectar la participación social y multicultural con los gobiernos locales y de regiones subnacionales, de modo de fortalecer la sociedad civil en esos ámbitos y reforzar la autonomía territorial, manteniendo el principio de subsidiariedad y la unidad del Estado. Esto involucra el uso de la comunicación electrónica para el libre acceso a la información pública, como medio de consulta, interacción y debate y para asegurar la igualdad de oportunidades. Con este fin será preciso ampliar la utilización comunitaria de los medios electrónicos y acrecentar los equipamientos y fondos públicos para facilitar el ejercicio de la participación democrática por los ciudadanos. Además, habrá que buscar formas innovadoras de entregar financiamiento público a las instituciones de la sociedad civil para fortalecer la participación y el ejercicio de la ciudadanía. Resulta fundamental en esta perspectiva el trabajo coordinado de la sociedad civil y del Estado en función del bien común. Sin embargo, la sociedad civil no puede ser confundida con un partido político o con una propuesta de poder; no puede tener una estrategia acabada de transformación social. Toda aspiración a copar el espacio público por un actor social o cultural o un proyecto político implica negar el espacio propio de la diversidad y la libertad, imprescindible para que la sociedad civil misma pueda expandirse. Es necesario reforzar la capacidad de autonomía de las personas y de la sociedad civil, como mejor defensa contra todo tipo de hegemonía o absolutismo. El desafío, entonces, consiste en comprender y fomentar la articulación de los diversos actores, intereses y orientaciones en nuevos espacios de deliberación y participación que generen una nueva cultura cívica y política y promuevan opciones de democracia y desarrollo.

iv) Aumentar la capacidad de acción del Estado. El mercado y el Estado, en una concepción renovada del desarrollo, pueden actuar de manera concomitante en función de la sociedad y la provisión de bienes públicos. Así lo han puesto de manifiesto recientemente los actores y las orientaciones renovadas de la política y el desarrollo. La reorganización y renovación del Estado deben considerar al menos tres aspectos:

- La inserción en los mercados globales se rige por el paradigma de competitividad sistémica; lo que pesa no es la competitividad de una u otra empresa, sino la capacidad de organización y gestión del país para articular factores económicos y no económicos. La competitividad implica un rol coordinador del Estado en los distintos procesos de competitividad sistémica.

- El peso del mercado y sus tendencias disgregadoras otorgan un rol primordial al Estado como instancia para asegurar la cohesión social. La reformulación del Estado de bienestar y de las políticas de desarrollo es un paso clave para hacer viable el desarrollo. 
- El Estado de derecho comprende la acción ciudadana autónoma, ya que, en suma, es un Estado democrático capaz de expresar y cohesionar a la comunidad ciudadana. Resulta indispensable incrementar la capacidad de acción de un Estado legítimo que pueda participar en la globalización en nombre de la sociedad y en relación constante con ella. La capacidad de cohesión social del Estado permitirá, de acuerdo con los intereses de la nación, vincular el crecimiento económico, las políticas distributivas y las políticas de desarrollo para mejorar la justicia social y reducir la pobreza. Es fundamental regenerar las instituciones del Estado, lo que supone a la vez fortalecer una sociedad autónoma del Estado que solo puede ser definida en cada país según su situación histórica particular. En síntesis, parece esencial fortalecer la capacidad y legitimidad del Estado para asumir y desempeñar eficazmente la función de agente del desarrollo y de la democracia. Esta "estatalidad", más allá de la función de asistencia técnica, puede traducirse en la integralidad de los programas nacionales a partir de una visión agregada e intersectorial; la necesidad de coordinación a través de áreas programáticas específicas y de jurisdicciones institucionales; la incorporación de mecanismos de transparencia en la gestión y también de responsabilidad del poder político y de los actores privados y las organizaciones de la sociedad civil; la articulación público-privada en el desarrollo, con la puesta en práctica de programas y proyectos, y la adaptación de políticas e instrumentos a las diferentes realidades nacionales y a los retos planteados por la relación nación-municipios.

v) Asociar los avances en el plano de la equidad con el fortalecimiento y la renovación de las instituciones de la democracia. Es imprescindible la interacción virtuosa entre equidad e institucionalidad, en función de valores y metas sociales comunes. La equidad supone primordialmente el reconocimiento práctico de la igualdad de todos y la eliminación definitiva de prácticas estamentales y de clientelismo, tan comunes en la vida política de América Latina. Es importante promover una cultura política práctica asociada con mecanismos de sanción institucional para la discriminación étnica, de género, de migrantes y de otros grupos minoritarios. La equidad no solamente implica corregir una distribución desigual sino también considerar las demandas y disposición de los ciudadanos, con el propósito de reconocer y modificar situaciones de inequidad a las que están sometidos variados grupos sociales. En este sentido, el problema estructural de la pobreza y la desigualdad en América
Latina debe ser entendido como un problema político y encarado con medidas encaminadas a que los pobres y excluidos transformen sus carencias en demandas que sean recogidas por el sistema institucional.

vi) Fortalecer las capacidades de ciudadanos hoy más autónomos y activos. Es muy importante reforzar y legitimar la práctica de una ciudadanía activa y autónoma, con ciudadanos más informados, que arraigan mejor su autonomía y libertad y que tienen más capacidad de hablar y ser escuchados. Los partidos políticos ya no pueden imponerse sin escuchar, ni gobernar sin convencer, y deben responder cada vez más a exigencias de verdad, transparencia y respeto ciudadano. Ante el surgimiento y consolidación de una ciudadanía de esta índole y la apertura de nuevos espacios de deliberación política, se hace indispensable renovar los sistemas de representación y acción políticas.

vii) Aquilatar la importancia de los nuevos medios de comunicación en la configuración del espacio público. Las sociedades latinoamericanas están cada vez más interconectadas. Los ciudadanos se informan y adoptan sus posiciones en materias de interés público a partir de la deliberación que se da en espacios abiertos, especialmente a través de los medios de comunicación y las nuevas tecnologías de información. Los ciudadanos escuchan y se hacen oír, además, mediante la opinión pública y su nexo con los medios de comunicación. Si bien las tendencias al control privado de la comunicación pública son evidentes, parece fundamental que los ciudadanos desarrollen capacidades tecnológicas que les posibiliten nuevas formas de participación y comunicación a través de teléfonos celulares, blogs, Internet y otros medios. Las modernas tecnologías de la información y de las comunicaciones son poderosas fuerzas de un nuevo tipo de comunicación personal, participativa e interactiva. La comunicación electrónica puede facilitar la deliberación, la formación de opiniones y la difusión de ideas. Sería útil formular propuestas políticas que abran nuevas formas de participación de la ciudadanía y de fiscalización ciudadana sobre la acción de las instituciones públicas y que se sometan al debate público a través de estos sistemas de comunicación que penetran cada vez más en la vida cotidiana de las personas. El futuro de la gobernabilidad democrática está vinculado a la transformación de las esferas públicas tradicionales en sistemas de comunicación interactivos. Es difícil combatir la política del escándalo o la conspiración restringiendo a los medios de comunicación: lo que debe hacerse es fomentar la deliberación y disminuir la corrupción. Para alcanzar estos objetivos es esencial mejorar el 
acceso de los medios de comunicación a las fuentes de información sobre prácticas corruptas, para incidir en su disuasión; establecer un estatuto de protección del periodista, y adoptar, en conjunto con los medios de comunicación, medidas encaminadas a regular las acciones de estos medios.

\section{Bibliografía}

Arias, O. y S. Duryea (2003): Social Exclusion Due to Race or Ethnicity in Latin America: What Do We Know?, Working Paper, Washington, D.C., Banco Interamericano de Desarrollo.

Buvinic, M. y V. Roza (2004): Women, Politics and Democratic Prospects in Latin America, Sustainable Development Department Technical Papers Series, wID 108. Disponible en http://www. iadb.org/sds/doc/women.pdf

CEPAL (Comisión Económica para América Latina y el Caribe) (2006a): Anuario estadístico de América Latina y el Caribe 2005, LC/G.2311-P, Santiago de Chile. Publicación de las Naciones Unidas, $\mathrm{N}^{\circ}$ de venta: E/S.06.II.G.1.

(2006b), Primer informe sobre proyecciones económicas para el PAPEP, Santiago de Chile.

(2007): Panorama social de América Latina 2006, LC/G.2326-P, Santiago de Chile. Publicación de las Naciones Unidas, $\mathrm{N}^{\circ}$ de venta: S.06.II.G.133.

Corporación Latinobarómetro (2005): Informe Latinobarómetro 2005 , Santiago de Chile. Disponible en: http://www.latinobarometro. org/uploads/media/2006_01.pdf

(2006): Informe Latinobarómetro 2006, Santiago de Chile. Disponible en: http://www.latinobarometro.org/uploads/ media/2006_01.pdf

Duryea, S. y M.E. Genoni (2004): Ethnicity, race and gender in Latin American labor markets, en M. Buvinic, J. Mazza y R. Deutsch (comps.), Social Inclusion and Economic Development in Latin
America, Washington, D.C., Banco Interamericano de Desarrollo/ Johns Hopkins Press.

González, L.E. (2006): Las crisis políticas de América Latina en los primeros años del siglo, en D. Achard y L.E. González (comps.), Política y desarrollo en Honduras, 2006-2009, Tegucigalpa, Programa de las Naciones Unidas para el Desarrollo (PNUD).

Moreira, C. (2006): De la "Suiza de América" al "paisito": escenarios y prospectivas del Uruguay en la tercera ola de la democracia, PAPEP-PNUD Uruguay.

Observatorio Regional PAPEP (2006-2007): Situación actual y perspectivas de América Latina, según elites político-profesionales de EE.UU., elites europeas y elites de Argentina, Brasil y México, PAPEP-PNUD.

PNUD (Programa de las Naciones Unidas para el Desarrollo) (2004): La democracia en América Latina. Hacia una democracia de ciudadanas y ciudadanos, Buenos Aires, Aguilar/Altea-Taurus/ Alfaguara.

PNUD Argentina (2005): Ronda de consultas y encuesta de opinión pública, Buenos Aires, PAPEP-PNUD Argentina.

PNUD Bolivia (2004): Informe nacional de desarrollo humano en Bolivia 2004. Interculturalismo y globalización: la Bolivia posible, La Paz, Plural.

PNUD Honduras (2005): Honduras: los acuerdos son posibles. La experiencia de la Comisión Política de los Partidos Políticos, Tegucigalpa, Editorial Guaymuras. 